\title{
Punción ecoguiada frente a la punción ciega en el bloqueo nervioso del pie: revisión bibliográfica narrativa
}

Ecoguided puncture in front of the blind puncture in the nervous lock of the foot: narrative bibliographical review

Cristina Mora Castillo

Departamento de Podología, Universidad de Barcelona, L'Hospitalet de Llobregat, Barcelona. España

Palabras clave:

Anestésico local, bloqueo nervioso, efectividad, punción "ciega", punción guiada.

\section{Resumen}

Introducción: La punción guiada es una técnica utilizada para el bloqueo nervioso del pie, permitiendo visualizar estructuras anatómicas y la aguja de punción, consiguiendo así disminuir la cantidad de anestésico local sin dañar las estructuras. El objetivo de esta revisión es valorar la aplicación de esta técnica en el pie, realizando una revisión bibliográfica comparativa frente la punción a ciegas valorando la efectividad de ambas técnicas.

Material y métodos: Se ha realizado una revisión bibliográfica en las bases de datos más relevantes, incluyendo veintiocho artículos hasta septiembre de 2019. Las variables estudiadas fueron la cantidad de anestésico local, así como el dolor postoperatorio de ambas técnicas. Finalmente, se incluyen diez artículos para su revisión.

Resultados: Los artículos indican una disminución de la cantidad de anestésico local en el bloqueo de tobillo y ser más efectivo en la escala definida con esta técnica.

Discusión: La punción guiada es una técnica es más efectiva, sencilla de utilizar y podemos evitar bloqueos fallidos.
Keywords:

Local anaesthesic, nerve block, effectiveness, blind puncture, guided puncture.

\section{Abstract}

Introduction: Guided puncture is a technique used for the nerve block of the foot allowing to visualize anatomical structures and the puncture needle, thus reducing the amount of local anesthetic without damaging the structures. The objective of this review is to evaluate the application of this technique in the foot by carrying out a comparative study to the blind puncture.

Material and methods: A review of the literature has performed in the most relevant databases including twenty eight articles until Septembre 2019. The variables studied were the amount of local anesthetic as well as the postoperative pain of both techniques. Finally, ten articles are included for review.

Results: Articles indicate a decrease in the amount of local anesthetic into the ankle lock and be more effect on the scale defined with this technique.

Discussion: Guided puncture is a more effective, easy to use and we can avoid failed blockades. 


\section{INTRODUCCIÓN}

La punción guiada es una técnica que permite visualizar, en tiempo real, movimiento y posición de la aguja de punción hasta la estructura deseada con el fin de introducir y distribuir la adecuada cantidad de anestésico local. Mediante esta técnica se pretende evitar la punción "ciega" para la localización nerviosa ${ }^{1-3}$.

Actualmente, la anestesia guiada por ultrasonido ha sido rápidamente adoptada por los anestesiólogos, proporcionando los medios adecuados para identificar y bloquear de manera fiable los nervios periféricos ${ }^{1,2,4,5}$. Diversos estudios demuestran que este tipo de anestesia supone una reducción del tiempo de inicio del procedimiento quirúrgico ${ }^{6-8}$, incrementando así la tasa de éxito ${ }^{7-11}$ con menores dosis de anestésico local y, por consecuencia, un bloqueo más rápido ${ }^{4,10,12}$.

La principal ventaja es la disposición óptima del anestésico local lo suficientemente cerca del nervio, asegurando así un inicio rápido pero preservando al mismo tiempo las estructuras neurales y minimizando el daño a los tejidos adyacentes $^{8,11}$. Para el bloqueo nervioso se utilizan anestésicos locales de tipo amida ${ }^{12-14}$ (como lidocaína, mepivacaína, bupivacaína, ropivacaína, etc.) por su larga duración postoperatoria y prolongación en el bloqueo quirúrgico ${ }^{13,14}$.

Para la realización de esta técnica existen dos formas de punción: punción en plano o punción fuera de plano 1,12,15. La punción en plano permite visualizar la aguja a medida que la vamos introduciendo y, por lo tanto, en toda su longitud. En cambio, la punción fuera de plano es más compleja y busca visualizar únicamente la punta de la aguja'. Cualquiera de las dos modalidades de punción puede resultar útil. Elegir una forma u otra varía dependiendo de la anatomía específica del

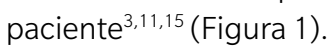

1
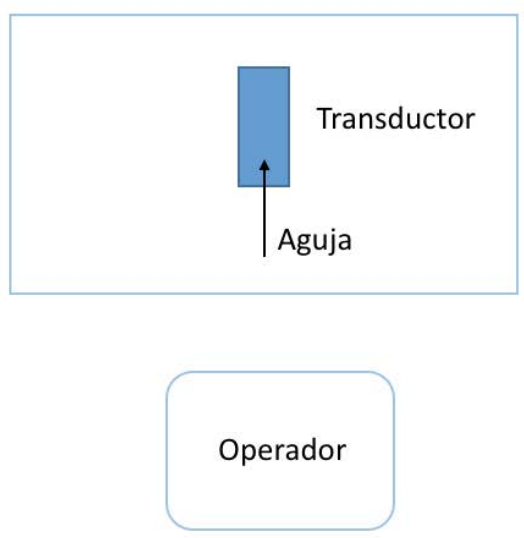

Figura 1. 1) Punción en plano. 2) Punción fuera de plano.

\section{1}

A nivel de la extremidad inferior, este método de anestesia de tobillo y pie ha demostrado ser más preciso que la punción "ciega"16. Cunnington y cols. ${ }^{16}$ demostraron en su estudio que las punciones guiadas por ultrasonido eran más precisas en manos de una persona inexperta que una punción "ciega" por un profesional experimentado. Por lo tanto, es una técnica que demuestra seguridad y eficacia ${ }^{8,11}$.

A pesar de que existen estudios que han demostrado que la punción ecoguiada en bloqueos del miembro inferior es superior, el propósito de este artículo es valorar la efectividad, concretamente en el bloqueo nervioso del pie y tobillo mediante una revisión bibliográfica.

\section{MATERIAL Y MÉTODOS}

Para la realización de este trabajo se realizó una búsqueda exhaustiva de revisión bibliográfica como base de datos principal PubMed. La búsqueda se completó con las bases de datos Science Direct, Dialnet y Revista Española de Anestesiología y Reanimación hasta septiembre de 2019. Las palabras claves utilizadas fueron ["ultrasound-guided AND ankle"], ["ultrasound-guided AND foot"], ["nerve block AND guided AND foot"], ["anesthesia AND foot"], ["punción guiada AND pie"] y ["anestesia AND pie"].

Se realizó una búsqueda donde se incluyeron todos los artículos de revisión y ensayos clínicos que hablaran de la punción guiada en el pie a la hora de realizar la anestesia. En la segunda búsqueda se incluyeron también todos los artículos de revisión y ensayos clínicos que hablaran de la anestesia en el pie. En ambas búsquedas se limitó que los artículos fueran publicados en los últimos 5 años y en paciente humanos adultos en los cuales se realizó un blo-

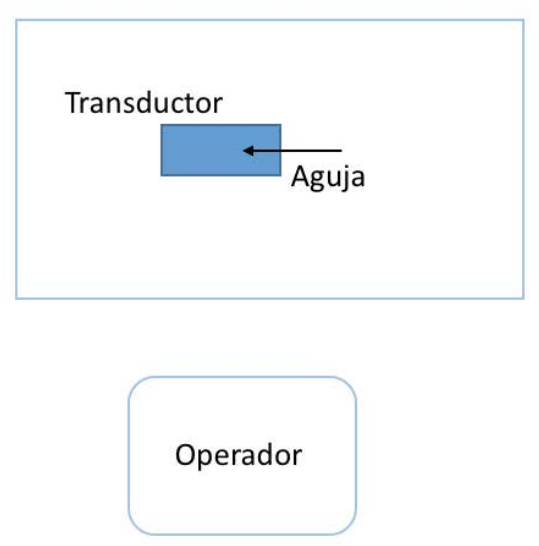

[Rev Esp Podol. 2019;30(2):101-108] 


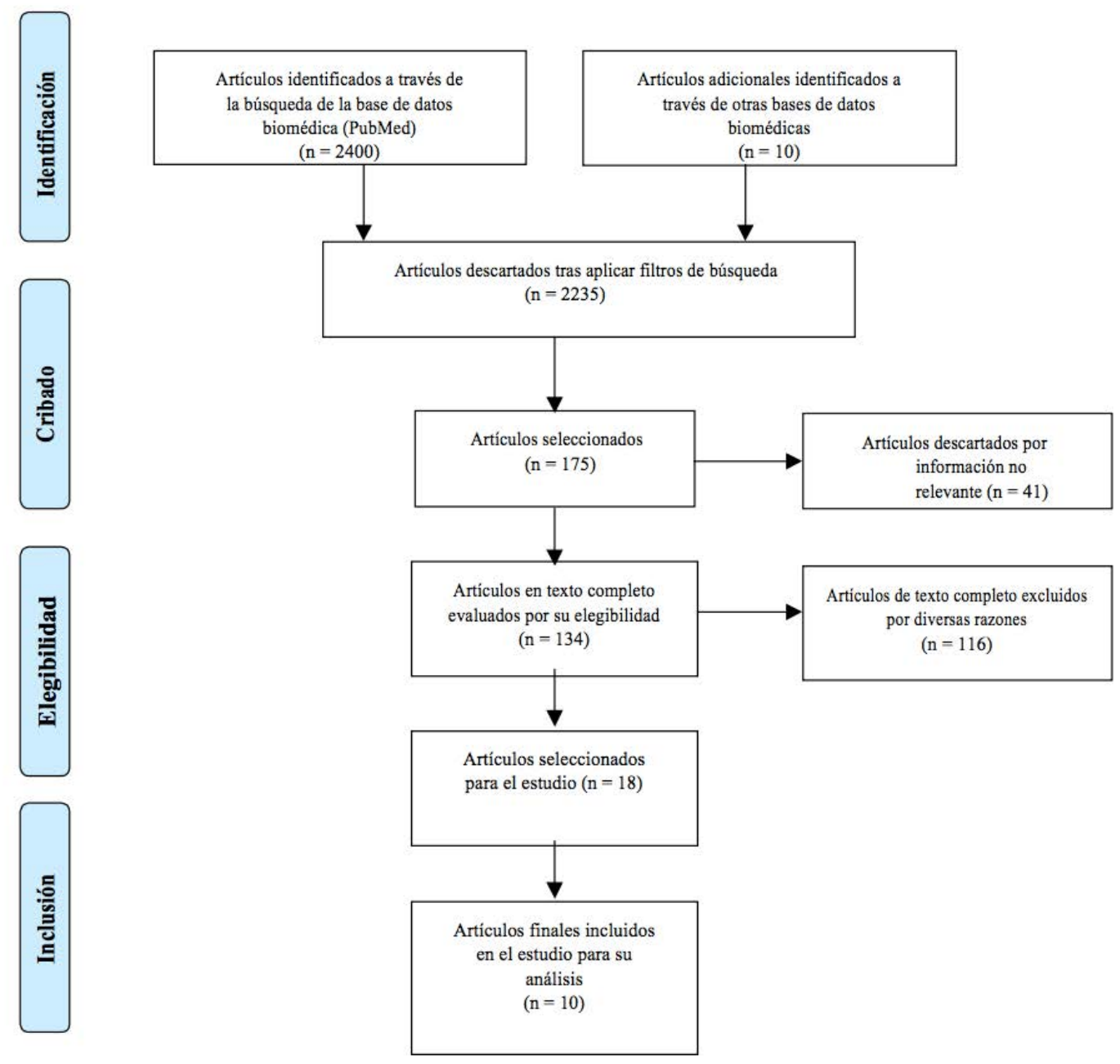

Figura 2. Diagrama de Prisma donde se muestra el procedimiento de selección de artículos.

queo a nivel de tobillo. Además, se acotó la búsqueda en inglés y español. Posteriormente, se amplió la búsqueda a los últimos 10 años de antigüedad. Se excluyeron todos los artículos que realizaran un bloqueo a nivel de hueco poplíteo y bloqueo de nervio ciático para anestesiar el pie.

La Figura 2 presenta el flujo de artículos utilizados en la presente revisión bibliográfica.

Un total de 2410 artículos fueron identificados inicialmente. Tras aplicar los filtros de cribado, 2253 fueron descartados por no estar relacionados con el trabajo. De los 175 artículos restantes, fueron descartados 41 por información no relevante. Se procedió a la lectura de los resúmenes de 134 artículos y descartaron 116 por no cumplir con los criterios de inclusión. Finalmente, fueron seleccionados 18 artículos. Posteriormente se seleccionaron 10 de ellos (de los 18 iniciales) para ser analizados debido a que se adaptan a la características del trabajo.
La cantidad de anestésico local, el tipo de anestésico, así como el dolor postoperatorio de ambas técnicas fueron las variables principales a estudiar.

Esta selección se llevó a cabo mediante la lectura del título y del abstract.

Un solo revisor, y siempre el mismo (CMC), llevó a cabo la selección de los artículos y el análisis crítico de los mismos.

\section{RESULTADOS}

Tras realizar los filtros de cribado, un total de 1060 pacientes fueron incluidos en los diez artículos analizados 9,5,17-24. No se especificó como datos relevantes ni la edad media de los pacientes ni el sexo. Siete de los diez artículos utilizados ${ }^{5,9,20-24}$ realizan un bloqueo guiado, mientras que cuatro de ellos ${ }^{17-19,24}$ hacen un bloqueo a "ciegas". 
Siete de los diez artículos, ${ }^{5,9,17-20,24}$ utilizan anestésico local tipo amida, mientras que tres de los diez artículos ${ }^{21-23}$ utilizan anestésico local tipo éster.

La cantidad media de anestésico varía si el bloqueo es guiado o a ciegas. En siete de los diez artículos ${ }^{5,9,20-24}$ de bloqueo guiado se presenta una cantidad media de anestésico de 6,43 $\mathrm{ml}$ en contraposición del bloqueo a ciegas $17,18,19,24$, que presenta una cantidad media de anestésico de 10,25 ml. Otro punto a analizar, en la cantidad de anestésico, es si el bloqueo de tobillo es completo o presenta alguna variante en este bloqueo, sin especificar el tipo de punción. Cuando se realiza un bloqueo total de tobillo (safeno, tibial posterior y peroneos) $)^{9,17,18,24}$ existe una cantidad media de anestésico local de $13,25 \mathrm{ml}$. Cuando hay variantes, por ejemplo, safeno $0^{5,22}$ presenta una cantidad media de $3,33 \mathrm{ml}$, nervios digitales ${ }^{19,24}$ una cantidad media de $6,5 \mathrm{ml}$ y nervio plantar media ${ }^{20}$ una cantidad media de anestésico local de $7 \mathrm{ml}$.

Para realizar un estudio en profundidad, en cuanto a la cantidad de anestésico utilizado se ha discriminado entre artículos donde se realiza el bloqueo de uno o dos nervios del pie de aquellos en los cuales se realiza un bloqueo de todo el tobillo.

Si analizamos primero los artículos que realizan un bloqueo de uno o dos nervios del pi e, $^{5,18-24}$ encontramos unos valores que oscilan entre $1 \mathrm{ml}$ hasta los $10 \mathrm{ml}$ como máximo. Por lo tanto, en ambas técnicas existen bastantes semejanzas.

En cambio, cuando realizamos un bloqueo completo de todo el tobillo con punción guiada, como en el estudio de Chin y cols. $2012^{9}$, se inyectan de 5-8 ml. De distinta manera, en el estudio de Ramírez Gómez y cols. ${ }^{17}$ se realiza una punción a "ciegas", donde se inyectan de 10-15 ml para anestesiar tibial posterior y peroneos, además de $5-10 \mathrm{ml}$ para el safeno interno.

En dos de los diez artículos ${ }^{5,19}$ utilizan mepivacaína en combinación con levobupicaína o ropivacaína. En uno de ellos ${ }^{19}$ se utilizó la mepivacaína sola. También se empleó levopubicaína en uno de ellos ${ }^{24}$. En contraposición, en cuatro de los diez artículos ${ }^{9,17,18,20}$ utilizan mayoritariamente la lidocaína en combinación con bupivacaína, ropivacaína o levobupicaína. Por último, en tres de los diez artículos ${ }^{21-23}$ se usa cloroprocaína.

El tiempo de seguimiento de dolor posquirúrgico fue en ocho de los diez artículos ${ }^{17-24}$ estudiados de dos horas hasta la semana de seguimiento. Mientras que en dos de los diez ${ }^{5,9}$ no especificaba el tiempo de seguimiento. Este dolor posquirúrgico fue evaluado a través de escala de dolor. En cinco de los diez artículos ${ }^{5,21-24}$ de punción guiada se utiliza una escala definida, mientras que en dos de ellos ${ }^{9,20}$ no se especifica ninguna escala. En tres de los diez artículos ${ }^{17-19}$ de punción a "ciegas" se utiliza la escala visual analógica (EVA). En los estudios de punción guiada ${ }^{5,9,20-23}$ se demuestra una calidad de bloqueo superior a la punción a "ciegas", llegando a ser calificado de muy buena por los pacientes.

En las Tablas I y II se recogen los resultados de los diferentes estudios utilizados.

\section{DISCUSIÓN}

Para realizar un bloqueo nervioso en el pie se puede hacer de dos maneras: punción guiada o punción a "ciegas". Esta revisión ha sido realizada para determinar cuál de las dos técnicas es más efectiva, así como qué papel desempeña la punción guiada en el pie.

Para valorar la efectividad de ambas técnicas se han estudiado diferentes parámetros, entre ellos la anestesia. Los anestésicos locales más utilizados son los del grupo amida ${ }^{5,9,17-24}$. En los estudios ${ }^{5,9,17-24}$ podemos ver cómo indistintamente del tipo de punción se utilizan anestésicos de acción moderada-prolongada o anestésicos de acción breve, tanto de forma combinada como de forma aislada. En nuestro campo profesional, la utilización de combinación de anestésicos se utiliza para aumentar la duración del efecto y así conseguir disminuir el dolor postoperatorio ${ }^{18,19}$. La utilización de un anestésico $u$ otro y su tiempo de acción no es un dato relevante, ya que no existen diferencias significativas entre la punción guiada y la punción a "ciegas".

No se encuentran diferencias significativas en el tiempo de inicio entre el bloqueo de uno o dos nervios o completamente todo el tobillo. En ambas técnicas es un tiempo cercano a los 30 minutos $^{9,17,21-23}$. En dos de los diez artículos ${ }^{5,21}$ de punción guiada se consigue un inicio más rápido: de entre 10 y 20 minutos.

El segundo parámetro estudiado para comprobar la efectividad de las técnicas es el dolor postoperatorio. En los estudios de punción guiada ${ }^{5,21-24}$ utilizan una escala definida. A excepción de los de Chin y cols. $2012^{9}$ y Bruke y cols. $2016^{20}$ en los cuales no se especifica ninguna escala. En esta es valorada de (0-2) siendo 0 "ninguna sensación de dolor" y 2 "sensación normal". A excepción de Roberts y cols. ${ }^{24}$ que utiliza la EVA valorada de (0-10), considerando 0 "no dolor" y 10 "máximo dolor". Para los estudios de punción a "ciegas"17-19 se utiliza la EVA valorada de (0-10), considerando 0 "no dolor". En el menor de los casos un $61 \%$ de los pacientes ${ }^{21}$ así lo calificaron, en el mejor de los casos fue del $94 \%$. En ninguno de los estudios ${ }^{5,9,20-23}$ se especificó si hubo dolor postoperatorio o si fue necesario el uso de analgesia de rescate.

En cuanto a los artículos de punción a "ciegas" encontramos en el estudio Ramírez Gómez y cols. ${ }^{13}$ que la anestesia fue efectiva al $100 \%$, pero los pacientes tuvieron un dolor postoperatorio de 4.3 media en la escala EVA y, por lo tanto, precisaron de analgesia de rescate. De igual manera, en el estudio Martín y cols. ${ }^{18}$, se precisó esta analgesia en el $42 \%$ de los pacientes. A excepción de Roberts y cols. ${ }^{24}$, quienes en su estudio comparativo de ambas técnicas demostraron que no hubo diferencias estadísticamente significativas en cuanto a dolor ni al tiempo necesario de sensación normal del pie $(p<0.05)$. En este caso, todos los pacientes tenían una prescripción estandarizada de analgesia postoperatoria. 


\section{Tabla I. Estudios de cantidad de anestésico local para punción guiada y punción "a ciegas".}

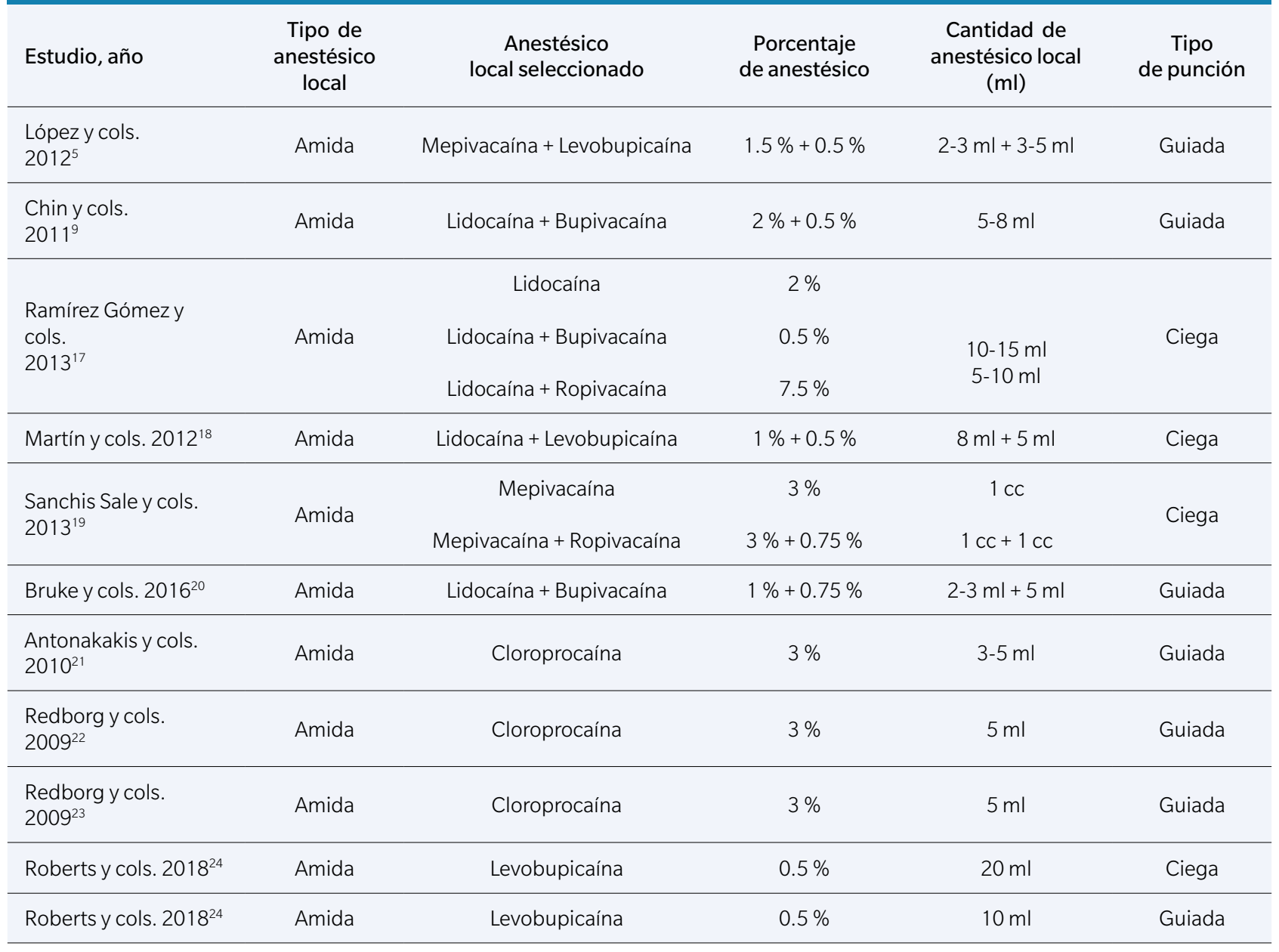

Respecto a las ventajas e inconvenientes de ambas técnicas, los autores concuerdan que la punción guiada da la posibilidad de identificar estructuras anatómicas en tiempo real, permitiendo de esta manera la visualización directa del nervio. Con esto conseguimos una disposición y distribución precisa del anestésico local alrededor de la estructura nervio$\mathrm{sa}^{1,15,20,24}$. Esta técnica, comparada con la técnica convencional, mejora significativamente la tasa de éxito, reduciendo la necesidad de menores dosis de anestésico local y reduciendo el número de analgesia postoperatoria ${ }^{1,9,24}$. Se trata de una técnica simple de bajo coste que permite realizar procedimientos invasivos, comparativos y dinámicos. Pudiendo realizar tratamientos selectivos como infiltraciones e incluso la liberación/descompresión efectiva de las estructuras responsables del síndrome del túnel tarsal ${ }^{25,26}$. En cambio, cuando realizamos una punción a "ciegas" existen diversas complicaciones: lesión nerviosa, toxicidad sistémica por anestésico local, hematomas y bloqueos fallido ${ }^{27}$.
López y cols. ${ }^{5}$ nos hablan de la importancia de la punción guiada a la hora de anestesiar ramas del nervio tibial y peroneo. Estas dos ramas nerviosas presentan múltiples variaciones anatómicas y frecuentes anastomosis entre ellas, demostrado en el estudio Bowness y cols. ${ }^{28}$, donde el nervio peroneo superficial responsable de la inervación dorsal del pie puede presentar variaciones anatómicas en el punto que perfora la fascia profunda. Esto puede contribuir a un bloqueo incompleto o fallido en el tobillo.

Por ello, intentar bloquear selectivamente estos nervios basándonos en referencias anatómicas puede resultar una anestesia desigual y conducir a bloqueos fallidos. Chin y cols. ${ }^{9}$ y Redborg y cols. ${ }^{22,23}$, en sus estudios comparativos entre ambas técnicas, demuestran un $84 \%$ y un $72 \%$, respectivamente, de éxito en la punción guiada. No obstante, la punción guiada como técnica de ultrasonido puede reducir, pero no eliminar, las complicaciones de la anestesia $^{1,24}$. 
Tabla II. Revisión bibliográfica de los diferentes parámetros biomédicos.

\begin{tabular}{|c|c|c|c|c|c|c|}
\hline Estudio, año & $\begin{array}{l}\text { Tipo de } \\
\text { punción }\end{array}$ & Muestra & Nervio & $\begin{array}{c}\text { Inicio } \\
\text { del bloqueo }\end{array}$ & $\begin{array}{c}\text { Tiempo } \\
\text { de seguimiento }\end{array}$ & Escala \\
\hline $\begin{array}{l}\text { López y cols. } \\
2012^{5}\end{array}$ & Guiada & $N=100$ & Safeno & $10 \mathrm{~min}$ & - & $\begin{array}{c}0-2 \\
\text { (2: sensación } \\
\text { normal, 0: } \\
\text { ninguna } \\
\text { sensación) }\end{array}$ \\
\hline $\begin{array}{l}\text { Chin y cols. } \\
2011^{9}\end{array}$ & Guiada & $N=518$ & $\begin{array}{l}\text { Tibial posterior, } \\
\text { peroneosy } \\
\text { safeno }\end{array}$ & $30 \mathrm{~min}$ & - & - \\
\hline $\begin{array}{l}\text { Ramírez Gómez y } \\
\text { cols. } 2013^{17}\end{array}$ & Ciega & $N=150$ & $\begin{array}{l}\text { Tibial posterior, } \\
\text { peroneos y } \\
\text { safeno interno }\end{array}$ & $30 \mathrm{~min}$ & $12-24 h$ & $\begin{array}{c}\text { EVA } \\
\text { (0-4, dolor } \\
\text { leve; 4-7, dolor } \\
\text { moderado; } 7-10, \\
\text { dolor severo) }\end{array}$ \\
\hline $\begin{array}{l}\text { Martín y cols. } \\
2012^{18}\end{array}$ & Ciega & $N=111$ & $\begin{array}{l}\text { Tibial posterior y } \\
\text { peroneos }\end{array}$ & $30 \mathrm{~min}$ & $8,12,24 \mathrm{~h}$ & $\begin{array}{c}\text { EVA } \\
(0-10, \\
0=\text { no dolor })\end{array}$ \\
\hline $\begin{array}{l}\text { Sanchis Sale y cols. } \\
2013^{19}\end{array}$ & Ciega & $N=64$ & $\begin{array}{l}\text { Digitales del 1. }{ }^{\text {er }} \\
\text { dedo }\end{array}$ & - & $\begin{array}{c}2,4,6,12,24 \\
48 h\end{array}$ & $\begin{array}{c}\text { EVA } \\
(0-10 \\
0=\text { no dolor })\end{array}$ \\
\hline $\begin{array}{l}\text { Bruke y cols. } \\
2016^{20}\end{array}$ & Guiada & $N=15$ & Plantar medial & - & $24 \mathrm{~h}$ & \\
\hline $\begin{array}{l}\text { Antonakakis y cols. } \\
2010^{21}\end{array}$ & Guiada & $N=18$ & Peroneos & $20-30 \mathrm{~min}$ & 24 h-1 semana & $\begin{array}{c}0-2 \\
\text { (2: sensación } \\
\text { normal, 0: } \\
\text { ninguna } \\
\text { sensación) }\end{array}$ \\
\hline $\begin{array}{l}\text { Redborg y cols. } \\
2009^{22}\end{array}$ & Guiada & $N=18$ & Sural & $30 \mathrm{~min}$ & $24 \mathrm{~h}$ & $\begin{array}{c}0-2 \\
\text { (2: sensación } \\
\text { normal, 0: } \\
\text { ninguna } \\
\text { sensación) }\end{array}$ \\
\hline $\begin{array}{l}\text { Redborg y cols. } \\
2009^{23}\end{array}$ & Guiada & $N=18$ & Tibial & $30 \mathrm{~min}$ & $24 \mathrm{~h}$ & $\begin{array}{c}0-2 \\
\text { (2: sensación } \\
\text { normal, 0: } \\
\text { ninguna } \\
\text { sensación) }\end{array}$ \\
\hline $\begin{array}{l}\text { Roberts y cols. } \\
2018^{24}\end{array}$ & Guiada & $N=25$ & $\begin{array}{c}\text { Tibial posterior, } \\
\text { peroneos } \\
\text { y safeno }\end{array}$ & & 2,6 y $24 \mathrm{~h}$ & $\begin{array}{c}\text { EVA } \\
\text { (0-4, dolor } \\
\text { leve; 4-7, dolor } \\
\text { moderado; } 7-10, \\
\text { dolor severo) }\end{array}$ \\
\hline $\begin{array}{l}\text { Roberts y cols. } \\
2018^{24}\end{array}$ & Ciega & $N=23$ & $\begin{array}{l}\text { Digitales } \\
\text { del 1. }{ }^{\text {er }} \text { dedo }\end{array}$ & & 2,6 y $24 \mathrm{~h}$ & $\begin{array}{c}\text { EVA } \\
\text { (0-4, dolor } \\
\text { leve; 4-7, dolor } \\
\text { moderado; } 7-10, \\
\text { dolor severo) }\end{array}$ \\
\hline
\end{tabular}


El uso de una técnica de ultrasonido requiere formación para adquirir los conceptos necesarios para realizar un punción guiada: este debe tener conocimientos anatómicos y habilidades suficientes para alinear el transductor, aguja y pantalla en el mismo plano para el éxito del bloqueo nervioso ${ }^{1,24-26}$. Esto sería un caso de operador dependiente. Lo argumentado en el párrafo anterior está en contradicción con lo citado por el autor Cunnington y cols. en el artículo Sahler y cols. ${ }^{16}$.

Referente al objetivo general de este trabajo se puede apreciar cómo la técnica de punción guiada ${ }^{5,9,20-23,24}$ es utilizada para el bloqueo de grandes ramas nerviosas e incluso para el bloqueo completo de tobillo. Los estudios de lborra y cols. ${ }^{25} \mathrm{e}$ Iborra y cols. ${ }^{26}$, ambos estudios demuestran el uso de la punción guiada para el bloqueo de ramas más pequeñas como el nervio plantar medial para conseguir anestesiar el nervio tibial. Una técnica utilizada, actualmente, para la liberación del túnel tarsal, evitando así menos riesgos asociados a la cirugía convencional. En cambio, en el estudio Sanchis Sale y cols. ${ }^{19}$ de punción a "ciegas" realizan un bloqueo de los nervios digitales del primer dedo.

Después de valorar la efectividad de ambas técnicas y las indicaciones/contraindicaciones, se debería seguir trabajando en más estudios para ampliar la aplicación de la punción guiada en el pie, debido a que se considera insuficiente la cantidad de datos relevantes en la información recogida.

En conclusión, la punción guiada presenta buenos resultados, es sencilla de utilizar y con ella evitamos bloqueos fallidos por variaciones anatómicas.

\section{CONFLICTO DE INTERESES}

La autora declara que no existe ningún conflicto de intereses relevante en este artículo.

\section{FINANCIACIÓN}

No existen fuentes de financiación públicas o privadas en la realización del presente estudio.

\section{BIBLIOGRAFÍA}

1. Aguirre Ospina OD, González Maldonado JF, Ríos Medina AM. Ergonomía en los bloqueos nerviosos guiados por ultrasonografía. Rev Colomb Anestesiol. 2015;43(4):331-9. DOI: 10.1016/j.rca.2015.06.002.

2. Domingo Rufes T. Aplicación de la punción ecoguiada en el tratamiento del dolor crónico [tesis doctoral]. Barcelona: Departament de Patologia i Terapèutica Experimental, Universitat de Barcelona; 2015.

3. Domingo T, Mayoral V, Casals M, Serrano A, Miguel M, Sabaté A. La punción guiada con ultrasonido aplicada a una unidad de dolor crónico. Rev Esp Anestesiol Reanim. 2010;57(8):493-507. DOI: 10.1016/S00349356(10)70710-6.

4. Orebaugh SL, Kentor ML, Williams BA. Adverse outcomes associated with nerve stimulator-guided and ultrasound-guided peripheral nerve blocks by supervised trainees. Reg Anesth Pain Med. 2012;37(6):57782. DOI: 10.1097/AAP.0b013e318263d396.
5. López AM, Sala-Blanch X, Magaldi M, Poggio D, Asunción J, Franco CD. Ultrasound-guided ankle block for forefoot surgery: the contribution of the saphenous nerve. Reg Anesth Pain Med. 2012;37(5):554-7. DOI: 10.1097/AAP.0b013e3182611483.

6. Karaarslan S, Tekgül ZT, Simsek E, Turan M, Karaman Y, Kaya A, et al. Comparison between ultrasonography-guided popliteal sciatic nerve block and spinal anesthesia for hallux valgus repair. Foot Ankle Int. 2016;37(1):85-9. DOI: 10.1177/1071100715600285.

7. Lee LA, Posner KL, Kent CD, Domino KB. Complications associated with peripheral nerve blocks: lessons from the ASA closed claims project. Int Anesthesiol Clin. 2011;49(3):56-67. DOI: 10.1097/ AlA.0b013e31821a0294.

8. Maalouf D, Liu SS, Movahedi R, Goytizolo E, Memtsoudis SG, Yadeau $J T$, et al. Nerve stimulator versus ultrasound guidance for placement of popliteal catheters for foot and ankle surgery. J Clin Anesth. 2012;24(1):44-50. DOI: 10.1016/j.jclinane.2011.06.008.

9. Chin KJ, Wong NWY, James A, Macfarlane R, Chan VWS. Ultrasound-guided versus anatomic landmark-guided ankle blocks. Reg Anesth Pain Med. 2011;36(6):611-8. DOI: 10.1097/AAP.0b013e31822b1291.

10. Lam NC, Petersen TR, Gerstein NS, Yen T, Starr B, Mariano ER. A randomized clinical trial comparing the effectiveness of ultrasound guidance versus nerve stimulation for lateral popliteal-sciatic nerve blocks in obese patients. J Ultrasound Med. 2014;33(6):1057-63. DOI: 10.7863/ ultra.33.6.1057.

11. Perlas A, Wong P, Abdallah F, Hazrati LN, Tse C, Chan V. Ultrasound-guided popliteal block through a common paraneural sheath versus conventional injection. Reg Anesth Pain Med. 2013;38(3):218-25. DOI: 10.1097/AAP.0b013e31828db12f.

12. Sala-Blanch X, de Riva N, Carrera A, López AM, Hadzic A. Ultrasound-guided popliteal sciatic block with a single injection at the sciatic division results in faster block onset than the classical nerve stimulator technique. Anesth Analg. 2012;114(5):1121-7. DOI: 10.1213/ ANE.0b013e318248e1b3.

13. Williams BA, Hough KA, Tsui BY, Ibinson JW, Gold MS, Gebhart GF. Neurotoxicity of adjuvants used in perineural anesthesia and analgesia in comparison with ropivacaine. Reg Anesth Pain Med. 2011;36(3):25530. DOI: 10.1097/AAP.0b013e3182176f70.

14. Gadsden J, Shariat A, Hadzic A, Xu D, Patel V, Maliakal T. The sequence of administration of $1.5 \%$ mepivacaine and $0.5 \%$ bupivacaine does not affect latency of block onset or duration of analgesia in ultrasound-guided interscalene block. Anesth Analg. 2012;115(4):963-7. DOI: 10.1213/ ANE.0b013e318265ba9a.

15. Girón Arango L, Vásquez Sadder MI, Gónzalez Obregón MP, Gamero Fajardo CE. Bloqueo de tobillo guiado por ultrasonido: una técnica anestésica atractiva para cirugía de pie. Rev Colomb Anestesiolg. 2015;43(4):283-9. DOI: 10.1016(j.rca.2015.07.002.

16. Sahler CS, Spinner DA, Kirschner JS. Ultrasound-guided first metatarsophalangel joint injections: description of an in-plane, gel standoff technique in a cadaveric study. Foot Ankle Spec. 2013;6(4):303-6. DOI: 10.1177/1938640013493465

17. Ramírez Gómez M, Schlufter Stolberg RM, Chao Rueda MI. Eficacia anestésica de los bloqueos periféricos a nivel de tobillo en cirugía de pie. Rev Mexicana Anestesiolg. 2013;36(1):14-25.

18. Martín MA, Ollé G, PellejeroJA, Torruella R, Yuste M, Pou N. Comparación de la eficacia analgésica postoperatoria del bloqueo de los nervios tibial (a nivel de maléolo interno) y peroneo común con infiltración de la herida quirúrgica en cirugía ambulatoria del hallux valgus. Rev Esp Anestesiol Reanim. 2012;59(4):197-203. DOI: 10.1016/j.redar.2012.02.008

19. Sanchís Sales E, Arnés Rodríguez A, Chiva Miralles MJ. Ropivacaína en bloqueos digitales del pie: ensayo clínico randomizado. Rev Esp Podol. 2013;24(2):52-55.

20. Bruke CJ, Adler RS. Tibial nerve block using an ultrasound-guided inframalleolar medial plantar nerve perineural injection: a technical note. J Clin Ultrasound. 2016;45(3):134-7. DOI: 10.1002/jcu.22446.

21. Antonakakis JG, Scalzo DC, Jorgenson AS, Figg KK, Ting P, Zuo Z, et al. Ultrasound does not improve the success rate of a deep peroneal nerve block at the ankle. Reg Anesth Pain Med. 2010;35(2):217-21. DOI: 10.1097/aap.0b013e3181c75db1. 
22. Redborg KE, Sites BD, Chinn CD, Gallgher JD, Ball PA, Antonakakis $J G$, et al. Ultrasound improves the success rate of a sural nerve block at the ankle. Reg Anesth Pain Med. 2009;34(1):24-8. DOI: 10.1097/ AAP.0b013e3181933f09.

23. Redborg KE, Antonakakis JG, Beach ML, Chinn CD, Sites BD. Ultrasound improves the success rate of a tibial nerve block at the ankle. Reg Anesth Pain Med. 2009;34(3):256-60. DOI: 10.1097/AAP.0b013e3181a343a2.

24. Roberts VI, Aujla RS, Fombon FN, Singh $\mathrm{H}$, Bhatia M. Is regional ankle blockin conjunction with general anaesthesia for first ray surgery? A randomised controlled trial of ultrasound guided ankle block versus "blind" local infiltration, FootAnkle Surg. 2018;11(12):1268-7731. DOI: 10.1016/j.fas.2018.11.012.

25. Iborra A, Villanueva M, Barret LS, Rodríguez-Collazo E, Sanz P. Anatomic delineation of tarsal tunnel innervation via ultrasonography. J Ultrasound Med. 2019;38(8):2067-79. DOI: 10.1002/jum.14897.
26. Iborra A, Villanueva M, Barret LS, Rodríguez-Collazo E, Sanz P. Ultrasound-Guided release of the tibial nerve and its distal branches: a cadaveric study. JUltrasound Med. 2019;38(8):2067-79. DOI: 10.1002/ jum.14897.

27. Capurro J, Sforsini CD. Seguridad en anestesia loco-regional (ALR). Complicaciones de los bloqueos nerviosos periféricos. Simposio. 2012;70(1):101-12.

28. Bowness J, Turnbull K, Taylor A, Halcrow J, Chisholm F, Grant C, et al. Identifying the Emergence of the Superficial Peroneal Nerve through deep fascia on ultrasound by dissection: implications for regional anesthesia in foot and ankle surgery. Sinapse $10^{\text {th }}$ anual scientific meeting. 2018. Edinburrgh. Br J Anaesth. 2019. DOI: 10.1016/j. bja.2019.02.003. 